\title{
Relations between aging sensory/sensorimotor and cognitive functions
}

\author{
Karen Z.H. Li ${ }^{\mathrm{a}, *}$, Ulman Lindenberger ${ }^{\mathrm{b}}$ \\ ${ }^{a}$ Department of Psychology, Centre for Research in Human Development, Concordia University, 7141 Sherbrooke St West, Montréal, Que., Canada H4B 1R6 \\ ${ }^{\mathrm{b}}$ Developmental Psychology Unit, Saarland University, Building 1, Im Stadtwald, D-66123 Saarbrücken, Germany
}

Received 1 March 2002; accepted 15 June 2002

\begin{abstract}
Recent evidence is reviewed to examine relations among sensory, sensorimotor, and cognitive aging. Age-heterogeneous cross-sectional data sets show substantial covariation among sensory, sensorimotor and intellectual abilities, and an increase in covariation from adulthood to old and very old age. Recent longitudinal analyses suggest that changes in sensory and intellectual functioning are interrelated. Experimental studies investigate the interdependence between cognitive and sensory/sensorimotor aging by examining the effects of simulated sensory loss on cognitive performance, or the effects of cognitive load manipulations on sensory or motor performance. Generally, both types of manipulations hinder older adults' performance more than that of younger adults. Theoretically, the age-associated intensification of the links among sensory, sensorimotor and cognitive functions observed both correlationally and experimentally may point to (a) common causes influencing all three functions; (b) an increase in resource overlap, cross-domain resource competition, and compensatory tradeoffs; and (c) a combination of the two. Future research aiming at discerning the relative import of these possibilities would profit from an integration of experimental and correlational research strategies.
\end{abstract}

(C) 2002 Elsevier Science Ltd. All rights reserved.

Keywords: Aging; Cognitive decline; Sensory decline; Hearing; Vision; Balance; Walking

In this paper, we review evidence for the hypothesized increase of covariation or interdependence between sensor$\mathrm{y} /$ sensorimotor and cognitive abilities with advancing age. It is well documented that sensory and sensorimotor abilities decline in the course of normative aging [54,57]. As well, extensive coverage of the normative age-related differences, changes, and continuities in cognitive and intellectual abilities is widely available [10,21]. Rather than reviewing these bodies of literature, we will focus selectively on empirical and theoretical work that has addressed the linkages between these domains with respect to adult development. The relevant evidence can be divided into two broad segments: (a) correlational evidence using crosssectional and longitudinal analyses, and (b) experimental evidence of an increased interdependence between domains. The final portion of our review will be devoted to discussing possible theoretical accounts of the documented relationships.

\footnotetext{
* Corresponding author. Tel.: + 1-514-848-7542; fax: + 1-514-848-2815.

E-mail addresses: kli@vax2.concordia.ca (K.Z.H. Li),

lindenberger@mx.uni-saarland.de (U. Lindenberger).
}

\section{Correlations between sensory, sensorimotor, and cognitive abilities}

Observed relationships between intellectual and sensory functioning have been discussed at length in the past several decades [12,54]. More recently, lifespan developmentalist Paul Baltes proposed that whereas before and during middle adulthood, interindividual variability in cognitive status is driven largely by cultural and environmental factors, in late life, variability might be increasingly constrained by biologically-based factors [7]. Subsequent to this proposal, several large-scale cross-sectional and longitudinal studies have examined the age-related changes in relationship between intellectual and sensory/sensorimotor abilities [3-6,8,35,36,52].

Sensory and cognitive differences: cross-sectional results. One relevant data set comes from the Berlin aging study (BASE) [9], a multi-disciplinary longitudinal study from which cross-sectional analyses were performed [35]. Relations between intellectual and sensory functioning (near and far visual acuity, pure tone auditory acuity) were assessed across a randomly drawn subsample of 156 old and very old (70-103 years) adults. Measures of fluid 
intelligence (termed cognitive mechanics) included multiple tests of perceptual speed, reasoning, and memory; measures of crystallized intelligence (termed cognitive pragmatics) included multiple tests of knowledge and verbal fluency. Importantly, visual and auditory acuity accounted for $93.1 \%$ of the age-related variance in intelligence. The results were similar even after subjects with extremely poor sensory acuity were dropped from the analyses, ruling out spurious effects due to floor level performance.

Subsequent cross-sectional analyses of a larger portion of data from the BASE project ( $n=516$, aged $70-103$ years) showed similar results [36]: composite measures of hearing, vision, and balance-gait accounted for $64.5,74.5$, and $82.6 \%$ of the age-related variance in general intelligence, defined as a second-order factor comprising the common variance of perceptual speed, reasoning, memory, knowledge, and verbal fluency. Adding an age-continuous sample of younger and middle-aged adults (25-69 years), these researchers further examined the hypothesized age-related increase in covariation between sensory/sensorimotor and intellectual abilities [8]. As hypothesized, an age-related increase in shared variance was observed between sensory (vision and hearing) and intellectual abilities (speed, reasoning, memory, knowledge, and fluency).

These results were interpreted as evidence of an ageassociated increase in the link between sensory and intellectual abilities, and suggest that cognitive aging may be attributable to the deterioration of common neurological processes. Salthouse et al. [52] reached similar conclusions with three independent samples, using tests of near visual acuity, working memory, associative learning, and concept identification. Similarly, Li et al. [32] have added to this view by demonstrating a relationship between short-term fluctuations in walking ability and short-term verbal and spatial memory in a healthy sample of residents aged 64-86 years. The degree of sensorimotor fluctuation increased with age, and correlated negatively with level of cognitive performance, again suggesting a possible common factor such as neurological deterioration that takes on increasing importance with advancing adult age $[33,34]$. Other crosssectional correlational work also suggests relationships between sensorimotor or physical and cognitive performance levels. For example, Anstey et al. [3] report relationships between lower limb strength and cognitive function, and relate this finding to age-changes in central nervous system or physical fitness. This extends earlier work relating measures of grip strength to cognition [20,26].

Another large-scale project, the Australian longitudinal study of aging (ALSA), has generated findings directly relevant to the BASE project [4]. Using a population-based sample of 894 participants aged 70-98 years old, Anstey et al. [4] employed measures of sensory and cognitive performance similar to those of Lindenberger and Baltes [35], while adding an episodic memory component. Similar to the BASE results, Anstey et al. found that a common factor explained most of the shared variance among cognition, age, speed and sensory function. However, some unique effects remained among these factors, perhaps owing to differences in the two test batteries. Similarly, Christensen et al. [17] reported cross-sectional findings using cognitive, sensory (visual acuity), and less obviously cognitive factors (e.g. grip strength, apolipoprotein E) in very old adults (aged 77-99 years). While a common factor encompassing cognitive and physical variables was obtained, these authors also found that grip strength and visual acuity had strong direct relationships with age $[17,4,6]$.

A methodological caveat. Lindenberger and Pötter [39] formally analyzed the use of variance partitioning procedures applied to age-heterogeneous cross-sectional data sets as a means to investigate the dimensionality of cognitive aging. Quite frequently, such procedures are used to determine the extent to which age relations between two or more domains of functioning such as vision and cognition are 'shared' or 'unique'. Lindenberger and Pötter [39] showed that the relative magnitude of shared and unique age effects is influenced by the covariance structure of age-orthogonal variance components and the magnitude of mean age trends. Consequently, shared age effects may come about by superimposed but causally independent age influences (see also the Monte Carlo simulations in Ref. [1]). Hofer and Sliwinski [27,56] have offered a similar methodological critique, initiating a recent series of commentaries [see Gerontology 48 (1)]. There is general agreement that the analysis of cross-sectional covariance structures needs to be complemented by other research designs (e.g. longitudinal, experimental, single-subject) and research traditions (e.g. cognitive neuroscience).

Sensory and cognitive change: longitudinal findings. Recent longitudinal results emanating from the BASE suggest that sensory and cognitive changes are not independent of each other [24,37]. Using latent growth curve modeling (LGM), Lindenberger and Ghisletta [37] analyzed 6-year changes in a total of eleven cognitive and sensory tasks from the BASE project. Individual differences in change were substantially correlated, with a single factor of common change accounting for about half of the total variance in change. The two measures of visual acuity included in the analysis both showed significant loadings on this common change factor. Ghisletta and Lindenberger [24] used the multivariate dual change score model introduced by McArdle [43] to analyze 6-year lead-lag relations among a total of four variables, two sensory (near and far visual acuity) and two intellectual (perceptual speed). Over time, cognitive variables affected sensory variables and vice versa. These recent analyses appear to provide at least partial support for previous conclusions based on crosssectional data [35]. Other longitudinal studies report somewhat less consistent significant relations between sensory and cognitive changes [2,5].

Summary of correlational evidence. Cross-sectional evidence extending into very old age is suggestive of a 
close connection between cognitive, sensory, and sensorimotor aging, though some recent findings suggest a common factor plus additional independent factors [4,17]. Links across domains appear to increase with age, and are consistent with the assumption of a common set of causes affecting the neurological systems associated with each domain. However, for methodological reasons, crosssectional correlational evidence does not allow for strong tests between competing causal hypotheses about the underlying structure of age relations. Recent longitudinal evidence indicates that interindividual differences in intellectual and sensory change are moderately correlated in old and very old age. Generally, cross-domain correlations seem to be more pronounced for vision than for hearing. To the extent that mean age trends and interindividual differences around mean age trends reflect similar mechanisms, these findings suggest that at least some of the mechanisms underlying age changes in sensory and intellectual abilities are functionally related.

\section{Experimental evidence}

To complement the correlational findings and focus more closely on specific processes that may result in the growing interdependence between sensory/sensorimotor and cognitive aging, we turn now to experimental efforts to address the same issues.

Simulations of auditory and visual decline. A growing number of studies have compared young (typically in their second and third decades) and older adults (in their sixth and seventh decades) on measures of cognitive ability while systematically manipulating the level of sensory load. For example, Pichora-Fuller et al. [49] presented sentences auditorially to younger and older adults in the context of multi-speaker crowd noise. This and subsequent studies are notable for their rigorous control of individual differences in auditory acuity, using screening methods to select participants or to individually adjust the sound intensity levels used to present the experimental stimuli. Pichora-Fuller et al. [49] found that word recognition for the auditory information was compromised as a result of reductions in signalto-noise $(\mathrm{S} / \mathrm{N})$ ratios. Importantly, older adults were only able to perform comparably to younger listeners when $\mathrm{S} / \mathrm{N}$ ratios were substantially increased, and they utilized contextual cues more effectively than younger adults under high noise conditions. A similar conclusion was reached when sentences were visually presented to young and older adults under standard and visually noisy conditions $[22,58]$.

More recently, Murphy et al. [46] compared young and older adults on a test of paired-associate learning using auditory stimuli presented under quiet or noisy conditions. Although young and older adults recalled the most recently presented word pairs comparably, older adults scored significantly worse when recalling earlier word pairs, suggesting an age-related deficit in consolidation of information into long-term memory, and not simply a threshold problem. Interestingly, when young adults performed the same task under noisy conditions, their results closely resembled those of the older adults under quiet conditions. Under noisy conditions, older adults suffered a significant drop in memory performance. This result suggests that some proportion of the observed agerelated cognitive deficits may be attributed to sensory deficits. The authors propose that in old age, a greater demand on common resources results in increased interdependence between sensory and cognitive domains [53,54, 60-62]. Alternatively, the addition of background noise may be construed as more of a cognitive stressor than perceptual (i.e. requiring selective attention processes) although this point is arguable because some initial filtering already occurs at the peripheral level of hair cells [54].

Using a similar logic, Lindenberger et al. [40] tested middle-aged adults on a battery of intelligence tests either with simulated losses in auditory acuity (ear covers), visual acuity (goggles), or both. Despite the appropriate manipulation checks to demonstrate reduced sensory acuity, this middle-aged sample did not show a sizeable drop in cognitive performance. The authors concluded that although the peripheral aspects of sensation may not be as strongly tied to cognitive aging, more central aspects of neurological and sensory systems may be. Schneider and Pichora-Fuller [54] suggest that although the methods of simulation used may have addressed threshold changes, other aspects of vision and audition may have been sufficiently untouched, allowing participants to carry out cognitive operations. Another possibility is that the middle-aged participants were able to overcome the experimental reductions in stimulus quality because of their relatively intact cognitive status, whereas for old and very old adults, reduced cognitive and sensory proficiency would have an exacerbating effect consequently creating a double-jeopardy situation.

Dual-task methods. Whereas the foregoing description of experimental studies has focused on the impact of sensory load on cognitive performance, a growing body of literature has addressed the opposite case: the sensory or sensorimotor performance of older adults should be compromised more than that of younger adults when a cognitive load is added. A standard method for imposing greater cognitive load is to divide attention between two tasks, thus occupying cognitive resources. An age-related increase in performance costs due to dividing attention between two cognitive tasks is well-documented [44]. We focus here on examples of sensory or sensorimotor tasks paired with cognitive tasks.

Concerning the impact of divided attention on sensory processing, Sekuler et al. [55] tested a large cross section of adult observers (15-84 years) on a measure of peripheral visual processing: useful field of view (UFOV). UFOV was tested either alone or concurrently with a central letter identification task. While performance on the central task increased with age but showed little effect of divided 
attention, error rates on the UFOV task increased disproportionately when the central letter identification task was performed concurrently. The authors concluded that older adults' diminished UFOV is constrained by age differences in attentional capacity.

Considerably more work has been produced recently with respect to divided attention and sensorimotor, rather than sensory, performance. Again, the logic of these studies is to demonstrate a growing interdependence between cognitive and sensorimotor domains in old age, with the expectation that an attentional or cognitive load should disproportionately hinder the balance or locomotor performance of older adults compared to young. Research from the fields of kinesiology and rehabilitation medicine has adopted the dual-task paradigm from cognitive psychology to examine how specific aspects of walking [16] and balance control $[13,14,59]$ are compromised by the addition of a cognitive load [23]. For example, Brown et al. recently [14] tested balance recovery by measuring center of mass before and after perturbations on a moving platform. Under dualtask conditions, participants engaged in a concurrent counting backwards task. Whereas young and older adults displayed similar counting speeds before perturbations, older adults were differentially slowed in their counting speed during the recovery period. As well, the recovery strategy under dual-task conditions differed between groups. The impact of attentional load also appears to depend on the type of concurrent cognitive task used, however. For example, Brauer et al. [13] compared young, healthy older, and balance-impaired older adults on a postural recovery task performed concurrently with a verbal reaction time to tones task. Only the balance-impaired older adults showed significant effects of divided attention in terms of balance recovery times and verbal reaction times, while the healthy young and older groups performed comparably under full and divided attention conditions.

In the field of cognitive aging, a growing interest in the relationship between cognitive and sensorimotor aging has prompted sensorimotor dual-task research that emphasizes cognitive issues such as reduced attentional capacity [38, 42], or stimulus dependent interference effects [41]. In contrast to earlier studies, this literature has also examined more complex cognitive tasks such as walking while talking [29] or walking while memorizing words using mental imagery $[31,38]$.

A recent walking and memorizing study [38] was motivated by the link between sensorimotor and cognitive abilities observed in earlier correlational work [35]. Young and older adults were trained to perform a memorizing task and to walk quickly and accurately on either a simple or complex walking path. On multiple measures of memory and walking performance, older adults showed a greater drop in performance under dual-task conditions, relative to young adults. These results were replicated and extended [31] by incorporating more extensive training of each task and individualized manipulations of task difficulty. Partici- pants performed the walking and memorizing tasks concurrently under varying levels of difficulty. In all cases, older adults were able to maintain high levels of walking, but showed significant effects of divided attention in the cognitive domain (Fig. 1). While a strong relationship between cognitive and sensorimotor ability was demonstrated, the findings also relate to the issue of task priority, in that older adults appeared to be protecting walking performance at the expense of cognitive performance.

Recent work by Kemper et al. [29] underscores the issue of task priority: in this study, young and older adults spoke on topics of general knowledge while walking at a comfortable pace, finger tapping, or ignoring extraneous noise. Speech samples were analyzed in terms of grammatical complexity, sentence length, and content. Overall, older adults' talking performance showed minimal effects of divided attention. Conversely, younger adults were more sensitive to concurrent task demands, suggesting that whereas they may have performed to capacity limits, older adults adopted a conservative (simplified) speech register.

The discrepancies across studies in terms of attentional allocation and task priority could be due to variations in task familiarity, instructional emphasis (maximal versus comfortable performance levels), or task type (spatial, verbal). More systematic investigations are required to clarify the results. Nevertheless, the extant work points to an agerelated increase in cross-talk between cognitive and sensorimotor tasks, as would be expected given the correlational findings.

Summary of experimental evidence. When younger adults' cognitive performance approximates that of older adults' after imposing a sensory load such as decreasing S/N

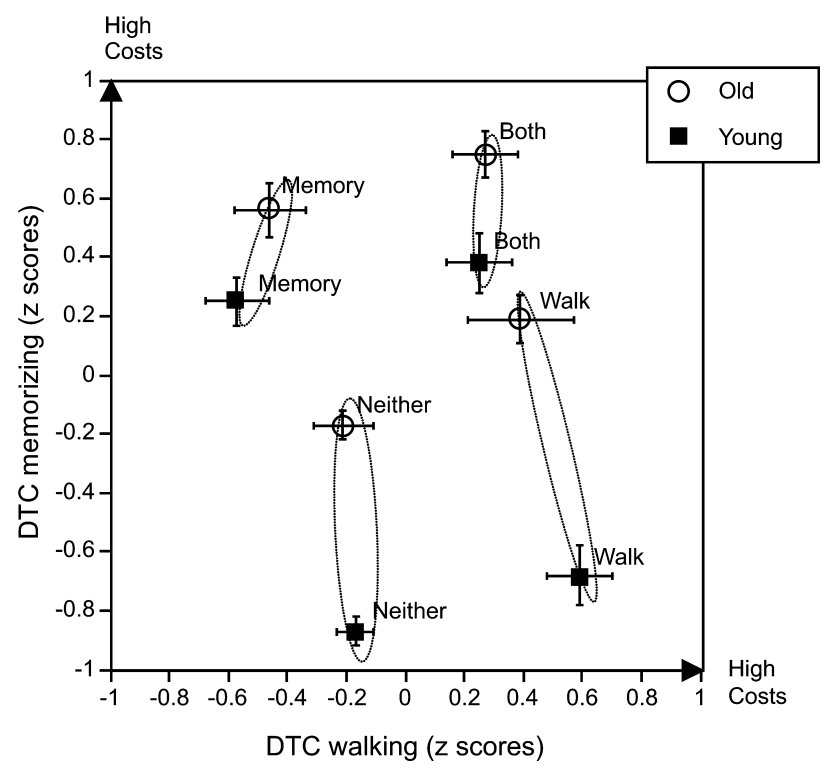

Fig. 1. Standardized dual-task costs for young and old adults during concurrent walking and memorizing. The four pairs of data points reflect four conditions in which the difficulty of the (a) memory, (b) walking, (c) memory and walking, and (d) neither domain was increased. Adapted from Ref. [31]. 
ratios, the majority of evidence supports the hypothesis that age-related cognitive declines are in part due to sensory declines. The experimental work involving concurrent or dual-task paradigms also points to an age-related increase in negative impact when sensory/sensorimotor tasks are performed concurrently with attention demanding cognitive tasks. Such results suggest an age-related increase in shared resources [54] or a compensatory reallocation of cognitive resources to protect balance, in some cases [31].

\section{Theoretical interpretations}

Together, the correlational and experimental findings provide convergent evidence for an increase in the link between sensory/sensorimotor and cognitive processes with advancing adult age. Understandably, the two areas of research and theorizing currently favor different yet compatible interpretations. On one hand, psychometric and computational modeling approaches appear to favor a common factor interpretation (see [33] for a review of theory and data). On the other hand, the experimental approach, with its orientation towards experimental control, quasi-experimental extreme groups (young versus old), and proximal explanation, favors an interpretation in terms of increasing reliance on common resources. When faced with increased challenge or declining ability, the sensorycognitive system seems to reorganize or reallocate resources to accommodate changing needs. While we acknowledge that these two theoretical perspectives are not incompatible, and arise from different research traditions, in the remaining discussion, we aim to evaluate each perspective in turn, and to consider ways in which extent and future research can be used to integrate these different levels of inquiry into a more coherent theoretical interpretation.

Common cause explanations. Within cognitive aging research, interest in evaluating the common cause hypothesis and related ideas has gained in momentum [4,17-19, 35]. The major premise of this hypothesis is that in late life, a common, biologically based factor is able to account for much of the age-related variance in sensory, sensorimotor, and intellectual functioning. Through computational modeling, Li et al. [33] have proposed a candidate common factor involving dopaminergic functioning and fluctuations in intraindividual levels of performance, for example. The related issue of increased covariation, or dedifferentiation, of intellectual factors with advanced age has met with mixed results, although only tentative conclusions are possible given the dearth of large longitudinal data sets $[18,19,24]$. In sum, common factor explanations of cognitive and sensory/ sensorimotor decline appear to converge in terms of finding substantial shared influences (i.e. a common factor), but appear to vary in terms of acknowledging unique influences associated with intellectual decline (i.e. does a common cause have to be the only cause?). It seems plausible to assume that common causes underly some but not all manifestations of sensory and cognitive change [35].

Resource reorganization explanations. In the majority of experimental studies reviewed, when either cognitive or sensory/sensorimotor processes are compromised through either age-related decline or experimental manipulation, substantial cross-domain effects are observed. In divided attention studies, the locus of the most substantial negative impact has been variable: in some cases the cognitive performance declines are differentially greater for older adults; in other cases the greater impact is imposed on sensory or sensorimotor processes. This variability is understandable, given the variety of tasks used, difficulty levels, and emphasis instructions. Across most studies, nonetheless, the negative impact appears to be greater for older than younger adults, suggesting competition for scarce resources and compensatory tradeoffs.

Given this broad agreement of findings, a plausible model of the relationship between sensory/sensorimotor and cognitive processes is a shared resources model [54]. In such a shared model, the increasing interdependence arises from progressive resource limitations and possible compensatory reallocation. For example, Pichora-Fuller et al. [49] observed in a speech-recognition study that older adults made more use of contextual cues than young adults when the auditory signal was degraded, thus utilizing cognitive processes to compensate for compromised sensory information. Similarly, Li et al. [31] observed older adults preserving high levels of walking performance while memorizing, but jeopardizing their cognitive performance to do so. Follow-up work with dementia patients and normal elderly control subjects indicates a similar bias towards balance maintenance [50].

Current findings from cognitive neuroimaging and aging provide some promising convergent evidence for the compensatory perspective. Cabeza's [15] hemispheric asymmetry reduction model (HAROLD) proposes an agerelated increase in bilateral brain activation in prefrontal cortex during cognitive tasks that are strongly lateralized in young adults [51]. Similarly, others have noted additional sites of activation in older compared to younger adults [25, 45], again suggesting neural recruitment or reorganization as a result of declines in primary neural pathways. While this rapidly growing area of research shows promise, more definitive evidence for compensatory change is required [48], and caution is needed when inferring age-differential processes on the basis of age-differential brain activation patterns [11].

A possible multi-level model. How is it possible to integrate the two classes of evidence reviewed here? The variety of methodology and levels of inquiry preclude any direct comparison. In addition, both the shared resource and common cause models, though plausible, are flexible and must be approached carefully to enhance falsifiability $[47,54]$.

We propose a multi-level approach, along the lines of 
that proposed by Li et al. [32,34], that attempts to delineate cause and effect more clearly. The onset of age-related cognitive and sensory declines occurs in early adulthood, and possibly sooner for sensory abilities [54]. While the specific locus of a common factor has yet to be confirmed, the resultant declines are likely to begin in an imperceptible way. Adaptations to functional loss may begin to occur in the form of neural reorganization and in the form of modifications to attentional allocation or processing strategy (e.g. invoking intact semantic processes to decode a degraded signal). It is unlikely that modifications of the latter sort are consciously controlled. Rather, the cognitive system of older individuals, as with those who have undergone acute losses, likely initiates behavior-level modifications that lead to more permanent neural circuitry changes. Paradoxically, this compensatory neural plasticity may be co-occurring with the central neural decline.

While this multi-level perspective does not solve the present challenge of refining either the common cause hypothesis or the shared resources perspective, we can at least outline a few directions for research along these lines. Most importantly, the onset of behavior-level modifications should be preceded by the onset of measurable cognitive and sensory declines. Critical to this will be an increased effort to assess the cognitive and sensory/sensorimotor status of individuals starting in midlife or earlier. Adjustments on the behavioral level, if observed, should then be followed by more permanent adjustments, possibly measurable in terms of neural recruitment or reorganization of circuitry. It remains to be seen whether the recently observed brain plasticity is a result of long-term microadjustments to behaviors, or if such recruitment phenomena occur flexibly in an on-line fashion that is determined by task demands.

This multi-level perspective on sensory, sensorimotor, and cognitive changes in aging also has methodological implications, as it puts much emphasis on the study of intraindividual change and variability [32]. To better study the sequence of events leading to age differences in 'functional cerebral space' [30] including loss-induced, behaviorally initiated neural plasticity, existing correlational and experimental designs should be complemented by multivariate, replicated, single-subject, repeated-measures designs in which individual subjects are followed over time in a variety of experimental conditions [28].

\section{Acknowledgements}

We gratefully acknowledge the research support to the first author from the Natural Sciences and Engineering Research Council of Canada (NSERC) and Fonds pour la Formation de Chercheurs et l'Aide à la recherche of Québec (FCAR), and to the second author from the Deutsche Forschungsgemeinschaft (DFG), Sonderforschungsbereich SFB 378, Project ARC (Aging, Resources, and Cognition).
We also thank Sarah Fraser for assisting with manuscript preparation.

\section{References}

[1] Allen PA, Hall RJ, Druley JA, Smith AF, Sanders RE, Murphy MD. How shared are age-related influences on cognitive and noncognitive variables? Psychol Aging 2001;16:532-49.

[2] Anstey KJ, Hofer SM, Luszcz MA. Trajectories of change in cognitive and sensory function: a latent growth curve analysis of unique and shared variance in rates of change and the effects of health and demographic predictors. Submitted for publication.

[3] Anstey KJ, Lord SR, Williams P. Strength in the lower limbs, visual contrast sensitivity, and simple reaction time predict cognition in older women. Psychol Aging 1997;12:137-44.

[4] Anstey KJ, Luszcz MA, Sanchez L. A re-evaluation of the common factor theory of shared variance among age, sensory function, and cognitive function in older adults. J Gerontol: Psychol Sci 2001;56B: P3-P11.

[5] Anstey KJ, Luszcz MA, Sanchez L. Two-year decline in vision but not hearing is associated with memory decline of very old adults in a population based sample. Gerontology 2001;47:289-93.

[6] Anstey KJ, Smith GA. Interrelationships among biological markers of aging, health, activity, acculturation, and cognitive performance in late adulthood. Psychol Aging 1999;14:605-18.

[7] Baltes PB. Prototypical paradigms and questions in life-span research on development and aging. Gerontology 1973;13:458-67.

[8] Baltes PB, Lindenberger U. Emergence of a powerful connection between sensory and cognitive functions across the adult life span: a new window to the study of cognitive aging? Psychol Aging 1997;12: $12-21$.

[9] Baltes PB, Mayer KU, editors. The Berlin aging study: aging from 70 to 100. New York: Cambridge University Press; 1999.

[10] Baltes PB, Staudinger UM, Lindenberger U. Lifespan psychology: theory and application to intellectual functioning. Ann Rev Psychol 1998;50:471-507.

[11] Bennett PJ, Sekuler AB, McIntosh AR, Della-Maggiore V. The effects of aging on visual memory: evidence for functional reorganization of cortical networks. Acta Psychol 2001;107:249-73.

[12] Birren JE, Botwinick J, Weiss AD, Morrison DF. Interrelations of mental and perceptual tests given to healthy elderly men. In: Birren JE, Butler RN, Greenhouse SW, Yarrow MR, editors. Human aging: a biological and behavioral study. Washington, DC: US Govt Printing Office; 1963. p. 143-56.

[13] Brauer SG, Woollacott M, Shumway-Cook A. The interacting effects of cognitive demand and recovery of postural stability in balanceimpaired elderly persons. J Gerontol: Med Sci 2001;56A:M489-96.

[14] Brown LA, Shumway-Cook A, Woollacott MH. Attentional demands and postural recovery: the effects of aging. J Gerontol: Med Sci 1999; 54A:M165-71.

[15] Cabeza R. Hemispheric asymmetry reduction in older adults: the HAROLD model. Psychol Aging 2002;17:85-100.

[16] Chen HC, Schultz AB, Ashton-Miller JA, Giordani B, Alexander NB, Guire KE. Stepping over obstacles: dividing attention impairs performance of old more than young adults. J Gerontol: Med Sci 1996;51A:M116-22.

[17] Christensen H, Mackinnon AJ, Korten A, Jorm AF. The common cause hypothesis of cognitive aging: evidence for not only a common factor but also specific associations of age with vision and grip strength in a cross-sectional analysis. Psychol Aging 2001;16: 588-99.

[18] Christensen H, Mackinnon AJ, Korten AE, Jorm AF, Henderson AS, Jacomb P. Dispersion in cognitive ability as a function of age: a 
longitudinal study of an elderly community sample. Aging NeuroPsychol Cogn 1999;6:214-28.

[19] Christensen H, Mackinnon AJ, Korten AE, Jorm AF, Henderson AS, Jacomb P, Rodgers B. An analysis of diversity in the cognitive performance of elderly community dwellers: individual differences in change scores as a function of age. Psychol Aging 1999;14:365-79.

[20] Clark JW. The aging dimension: a factorial analysis of individual differences with age on psychological and physiological measurement. J Gerontol 1960;29:423-9.

[21] Craik FIM, Salthouse TA, editors. The handbook of aging and cognition, 2nd ed. New Jersey: Erlbaum; 2000.

[22] Dickinson CM, Rabbitt PMA. Simulated visual impairment: effects on text comprehension and reading speed. Clin Vis Sci 1991;6:301-8.

[23] Ferrandez AM, Teasdale N, editors. Changes in sensory motor behavior in aging. Amsterdam: Elsevier; 1996.

[24] Ghisletta P, Lindenberger U. Exploring the structural dynamics of the link between sensory and cognitive functioning in old age: longitudinal evidence from the Berlin aging study. Submitted for publication.

[25] Grady CL, McIntosh AR, Horwitz B, Maisog JM, Ingerleider LG, Mentis MJ, Pietrini P, Schapiro MB. Age-related reductions in human recognition memory due to impaired encoding. Science 1995;269: $218-21$.

[26] Heron A, Chown S. Age and function. London: Churchill; 1967.

[27] Hofer S, Sliwinski M. Understanding aging: an evaluation of research designs for assessing the interdependence of aging-related changes. Gerontology 2001;47:341-52.

[28] Jones CJ, Nesselroade JR. Multivariate, replicated, single-subject, repeated measures designs and P-technique factor analysis: a review of intraindividual change studies. Exp Aging Res 1990;16:171-83.

[29] Kemper S, Herman RE, Lian CHT. The costs of doing two things at once for young and older adults: talking while walking, finger tapping, and ignoring noise or speech. Psychol Aging 2002; in press.

[30] Kinsbourne M, Hicks RE. Functional cerebral space: a model for overflow, transfer, and interference effects in human performance. In: Requin J, editor. Attention and performance VII. New Jersey: Erlbaum; 1978. p. 345-62.

[31] Li KZH, Lindenberger U, Freund AM, Baltes PB. Walking while memorizing: age-related differences in compensatory behavior. Psychol Sci 2001;12:230-7.

[32] Li S-C, Aggen SH, Nesselroade JR, Baltes PB. Short-term fluctuations in elderly people's sensorimotor functioning predict text and spatial memory performance: the MacArthur successful aging studies. J Gerontol 2001;47:100-16.

[33] Li S-C, Lindenberger U. Cross-level unification: a computational exploration of the link between deterioration of neurotransmitter systems and dedifferentiation of cognitive abilities in old age. In: Nilsson LG, Markowitsch HJ, editors. Cognitive neuroscience of memory. Seattle: Hogrefe and Huber; 1999. p. 103-46.

[34] Li S-C, Lindenberger U, Sikström S. Aging cognition: from neuromodulation to representation to cognition. Trends Cogn Sci 2001;5:479-86

[35] Lindenberger U, Baltes PB. Sensory functioning and intelligence in old age: a strong connection. Psychol Aging 1994;9:339-55.

[36] Lindenberger U, Baltes PB. Intellectual functioning in old and very old age: cross-sectional results from the Berlin aging study. Psychol Aging 1997;12:410-32.

[37] Lindenberger, U, Ghisletta, P. Cognitive and sensory changes in the Berlin aging study: longitudinal analyses of the total sample. Cognitive Aging Conference, Atlanta, GA; April 2002.

[38] Lindenberger U, Marsiske M, Baltes PB. Memorizing while walking: increase in dual-task costs from young adulthood to old age. Psychol Aging 2000;15:417-36.

[39] Lindenberger U, Pötter U. The complex nature of unique and shared effects in hierarchical linear regression: implications for developmental psychology. Psychiatr Meth 1998;3:218-30.

[40] Lindenberger U, Scherer H, Baltes PB. The strong connection between sensory and cognitive performance in old age: not due to sensory acuity reductions operating during cognitive assessment. Psychol Aging 2001;16:196-205.

[41] Maylor EA, Allison S, Wing AM. Effects of spatial and nonspatial cognitive activity on postural stability. Br J Psychol 2001;92:319-38.

[42] Maylor EA, Wing AM. Age differences in postural stability are increased by additional cognitive demands. J Gerontol: Psychol Sci 1996;51B:P143-54.

[43] McArdle JJ. A latent difference score approach to longitudinal dynamic structural analyses. In: Cudeck R, du Toit S, Sorbom D, editors. Structural equation modeling: present and future. Illinois: Scientific Software International; 2001. p. 342-80.

[44] McDowd JM, Shaw RJ. Attention and aging: a functional perspective. In: Craik FIM, Salthouse TA, editors. The handbook of aging and cognition, 2nd ed. New Jersey: Erlbaum; 2000. p. 221-92.

[45] McIntosh AR, Sekuler AB, Penpeci C, Rajah MN, Grady CL, Sekuler $R$, Bennett PJ. Recruitment of unique neural systems to support visual memory in normal aging. Current Biol 1999;9:1275-8.

[46] Murphy DR, Craik FIM, Li KZH, Schneider BA. Comparing the effects of aging and background noise on short-term memory performance. Psychol Aging 2000;15:323-34.

[47] Navon D. Resources—a theoretical soup stone? Psychol Rev 1984;91: 216-34.

[48] Park DC, Polk TA, Mikels JA, Taylor SF, Marshuetz C. Cerebral aging: integration of brain and behavioral models of cognitive function. Dialogue Clin Neurosci 2001;3:151-65.

[49] Pichora-Fuller MK, Schneider BA, Daneman M. How young and old adults listen to and remember speech in noise. J Acoust Soc Am 1995; 97:593-608.

[50] Rapp, M. Working memory and balance in older adults and patients with dementia. XVIIth World Congress of the International Association of Gerontology, Vancouver, BC; 2001.

[51] Reuter-Lorenz P, Jonides J, Smith ES, Harley A, Miller A, Marshuetz C, Koeppe RA. Age differences in the frontal lateralization of verbal and spatial working memory revealed by PET. J Cogn Neurosci 2000; 12:174-87.

[52] Salthouse TA, Hancock HE, Meinz EJ, Hambrick DZ. Interrelations of age, visual acuity, and cognitive functioning. J Gerontol: Psychol Sci 1996;51B:P317-30.

[53] Schneider BA, Daneman M, Murphy DR, Kwong-See S. Listening to discourse in distracting settings: the effects of aging. Psychol Aging 2000;15:110-25.

[54] Schneider BA, Pichora-Fuller MK. Implications of perceptual deterioration for cognitive aging research. In: Craik FIM, Salthouse TA, editors. The handbook of aging and cognition, 2nd ed. New Jersey: Erlbaum; 2000. p. 155-219.

[55] Sekuler AB, Bennett PJ, Mamelak M. Effects of aging on the useful field of view. Exp Aging Res 2000;26:103-20.

[56] Sliwinski M, Hofer S. How strong is the evidence for mediational hypotheses of age-related memory loss. Gerontology 1999;45:351-4.

[57] Stelmach GE, Hömberg V, editors. Sensorimotor impairment in the elderly. Dordrecht: Kluwer; 1993.

[58] Speranza F, Daneman M, Schneider BA. How aging affects the reading of words in noisy backgrounds. Psychol Aging 2000;15: $253-8$.

[59] Teasdale N, Bard C, LaRue J, Fleury M. On the cognitive penetrability of posture control. Exp Aging Res 1993;19:1-13.

[60] Tun PA, Wingfield A. One voice too many: adult age differences in language processing with different types of distracting sounds. J Gerontol: Psychol Sci 1999;54B:P317-27.

[61] Wingfield A, Lindfield KC, Goodglass H. Effects of age and hearing sensitivity on the use of prosodic information in spoken word recognition. J Speech Lang Hear Res 2000;43:915-25.

[62] Wingfield A, Tun PA, Koh CK, Rosen MJ. Regaining lost time: adult aging and the effect of time restoration on recall of time-compressed speech. Psychol Aging 1999;14:380-9. 